\title{
Erratum to: Differential expression of perilipin 2 and 5 in human skeletal muscle during aging and their association with atrophy-related genes
}

\author{
Maria Conte - Francesco Vasuri - Enrico Bertaggia - Andrea Armani - \\ Aurelia Santoro · Elena Bellavista - Alessio Degiovanni · Antonia D'Errico-Grigioni • \\ Giovanni Trisolino • Miriam Capri • Martino V. Franchi · Marco V. Narici • \\ Marco Sandri $\cdot$ Claudio Franceschi $\cdot$ Stefano Salvioli
}

Published online: 20 February 2015

(C) Springer Science+Business Media Dordrecht 2015

\section{Erratum to: Biogerontology \\ DOI 10.1007/s10522-014-9549-5}

The errors in the Fig. 2 caption and Table 1 of the original publication are corrected in this erratum.

1. In the legend related to Fig. 2, the text “ $\mathbf{j}$, $\mathbf{k}$ Ordinal logistic regression was applied to assess

The online version of the original article can be found under doi:10.1007/s10522-014-9549-5.

M. Conte $(\bowtie) \cdot$ A. Santoro - E. Bellavista · M. Capri ·

C. Franceschi · S. Salvioli

Department of Experimental, Diagnostic and Specialty Medicine and Interdepartmental Centre "L. Galvani"

(CIG), University of Bologna, via S. Giacomo 12,

40126 Bologna, Italy

e-mail: m.conte@unibo.it

F. Vasuri · A. Degiovanni - A. D'Errico-Grigioni Department of Experimental, Diagnostic and Specialty Medicine, S.Orsola-Malpighi Hospital, "F. Addarii" Institute of Oncology and Transplant Pathology,

University of Bologna, 40138 Bologna, Italy

E. Bertaggia · A. Armani · M. Sandri

Venetian Institute of Molecular Medicine, University of Padova, 35129 Padua, Italy the association between IHC score versus age" should be ignored.

2. In Table 1, the row headers "Plin2/graph" and "Plin5/graph" should be replaced with "Plin2/ gapdh" and "Plin5/gapdh".

E. Bertaggia $\cdot$ A. Armani · M. Sandri

Department of Biomedical Science, University of Padova, 35129 Padua, Italy

G. Trisolino

Reconstructive Hip and Knee Joint Surgery, Istituto

Ortopedico Rizzoli, 40136 Bologna, Italy

M. V. Franchi - M. V. Narici

Division of Clinical Physiology, School of Graduate Entry Medicine and Health, Derby Royal Hospital, University of Nottingham, Derby DE22 3DT, UK

M. Sandri

Telethon Institute of Genetics and Medicine, Pozzuoli, Naples, Italy 